Amelia RosselLI

Poesías

Trad. de Alessandra Merlo, con la colaboración de Juan Pablo Roa y Roberta Raffetto. Montblanc: Igitur, 2005

Antonella ANEDDA

Residencias invernales

Trad. de Emilio Coco. Montblanc: Igitur, 2005

Straziata figura di outsider in una penisola spesso occupata da officianti tecnocrati, colletti bianchi della lirica, burocratici e cauti amministratori di sé e del loro piccolo regno di carta, Amelia Rosselli, di dimora solo nella Caos-Cosmopolis del delirio idiomatico, rappresenta, senz'altro, nell'area linguistica italiana, insieme con il primo Antonio Porta e l'ultimo Volponi tra Con testo a fronte e Nel silenzio campale, il caso di più radicale applicazione del verbo surrealista, nelle sue componenti di scatenamento immaginoso, di resa al flusso tenebroso di un monologo oscuro. Proprio per questa adesione, senza ironie distanzianti o montaliane riserve e prudenze, ad una internazionale del lirico farneticamento, non cerebrale né tecnicistica, tantomeno programmatica, quanto abissalmente biologica, e necessaria, i suoi versi, paradossalmente, suonano meno stranieri, e di sé stupefatti, in castigliano che nell'orginale. Pronuncia assertiva e oracolare, sincopata e a singhiozzo ora, ora fluente in colate ritmiche di versi isosillabici e omoritmici, a perdifiato, suonano sorelle, anche per lo sgomento che le dettano, per gli umori civili in sobbalzo di un'ipersensibilità pura, alla Myschkin, del Poeta en Nueva York; ma nei raptus mistici, nella passionalità irrefrenata di un analogismo spinto, rimandano pure ad altre voci che il lettore italiano conosce grazie, davvero, a quella mirabile antologia che fu curata da Vittorio Bodini per gli einuadiani. Nella familiarità con cui ci giungono nella plenitudine sonora dello spagnolo le spasmodiche secrezioni viscero-mentali di questo monstrum lirico, (a volte biasci- cante per impercettibili ironie, a volte possente per voce d'amore, altre lieve di spiazzanti agudezas) conterà, e non poco, la ricerca rosselliana di un linguaggio universale nel quale «la lingua in cui scrivo volta a volta è una sola, mentre la mia esperienza sonora logica associativa è certamente quella di tutti i popoli, e riflettibile in tutte le lingue». Davvero è da salutare con gratitudine l'iniziativa presa da Igitur, dalle sue elegantemente spartane edizioni, di pubblicare una distillata antologia dell'esule mai esile scrittrice italiana, inedita nella penisola iberica (in lingua spagnola), non fosse per alcuni testi selezionati da Horacio Armani nella sua non dimenticabile e corposa antologia del Novecento lirico italiano, ora opportunamente ristampata dalla rivista Litoral.

Fantastica l'immaginoso lettore che all'ombra di questa operazione, nel dietro le quinte dei preliminari, vi sia la codirettice di collana Rosa Lentini, esperta della tumultuosa Pizarnik, di Rosa Leveroni, Perucho, de Ory, sapiente traduttrice di Pierre Reverdy e Djuna Barnes, promotrice di quella rivista, Hora de poesia (1979-1995) che anni or sono un attento volumetto monografico dedicó alla lirica italiana con traduzioni di testi di Caproni, Bertolucci, Sereni, tra gli altri. Austera e decantata, Lentini è lirica di scarne raccolte (La noche es una voz soñada, El sur hacia mí, El veneno y la piedra) pubblicate tra il 1994 e l'anno in corso, per i tipi di Icaria. Il titolo della collana che dirige questa strenua fedele dei versi, De Umbral en Umbral, sintagma nebbiosamente incoativo raccolto da un verso celaniano, a sua volta, forse, memore del 
Rilke de Le elegie duinesi, si adatta bene ad accogliere gli estremismi rimuginanti e ossessi, sempre sul limitare di una qualche imminenza, di Rosselli. A introdurla, però, è forse fuorviante la scelta dello storico intervento di Pasolini, che, se serví nel 1963, dal magnetofono de Il Menabò einaudiano, il sesto, a richiamare l'attenzione sull'eccentricità talentuosa e tenebrosa della voce di Rosselli, ora è da rileggere con guardinga cautela, un po' per i consustanziali difetti della critica pasoliniana, a volte sfuocata dall'accecamento viscerale, dal soffocante abbraccio fraterno, un po' per l'ovvia constatazione che quelle pagine potettero poggiare le proprie rapite argomentazioni su di un ristretto numero di versi, ristretto poi rispetto alla copiosa e diversa vena successiva e plurilingue dell'autrice: il lapsus, o la deviante erranza dalla grammatica costituiscono, come hanno notato Mengaldo, Fortini, Testa, un fenomeno dalla progressiva rarefazione nel corso di una traiettoria lirica, all'interno della quale la curatrice, Alessandra Merlo, seleziona campioni da Variazioni belliche, Serie Ospedaliera, Documento, e le verginali Poesie. Il prologo all'elefante garzantiano di un maestro come Giovanni Giudici, era forse un viatico più didascalico e ragionevole di quello genialmente rutilante di Pier Paolo Pasolini. Ma sono quisquilie. Veniamo alla traduzione di Merlo, muscolare ed elastica nel confronto con il chiuso ardore del dettato degli originali, psichicamente resistente alle scuoiate, diacerate, invasioni rosselliane. Di una versione, in qualche modo, normalizzante si tratta, comunque, grammaticalizzante e razionalizzante, senza dubbio. Prova ne siano proprio i versi d'avvio, «Y la alborada será/ese hilo de perlas que siempre llevas desabrochado/sobre tu pérleo/cuello, demacrado", che regolarizzano i più ellittici, e smozzicati d'affanno ma in una curva sintattica più sinuosa e distesa, «E l'albeggiare sarà/quella fila di perle tu porti ogn'ora slacciato sul tuo perleo/collo sma- grito»; ma pure la resa «Tú no sabes qué oscuro precipicio/ahumó mis ojos a tu/presencia:» di un dettato, tutto in minuscolo, «tu non sai quale oscuro precipizio/affumicò miei occhi a tua/vista», dove per la loro assenza brillano gli articoli: forse con un'anastrofe in chiasmo qualcosa dell'atterrita solennità del testo si sarebbe salvata. Inoltre: il verso in explicit in cui una lievemente subdola stortura suffissale (francesismo o anglismo?) fa capolino, "La congenitale tendenza al bene si risvegliava», è fatto più casto e roussoiano, diremmo, dalla scelta del traduttore, così, «La congénita tendencia al bien volvía a despertarse». Frugando qua e là troveremo che: "denta» e "doblezza», tra latineggiare e spagnoleggiare, neologismo e alterazione formativa della versione riprodotta dell'originale, vengono resi con «dientes " e "debilidad», ma il secondo è un refuso impunito per «debolezza», ed è forse un peccato; «lavata di testa», sintagma metaforico viene letteralizzato in "enjuague de cabeza», ma forse non importa nel contesto di un discorso che presentifica l'invisibile, astrae e concretizza, in continue psicomachie, anche cosmi-ironiche. Inevitabilmente qualcosa si perde sul piano del registro sonoro, dentro il quale la Rosselli signoreggia con lusso, di tutte le latitanze di annominationes, paranomasie, alliterazioni, omoteleuti, spesso argute e di sovrana ironia, veri wits alla Donne quelle che si rimpiangono davvero, per lo spazio metalinguistico in cui si ritrovavano, sono, nella riscrittura (alla Paolo Conte) di un celebre Rimbaud, le sillabe rabbrividite e tremolanti che segnalo in corsivo: «L'alba si presentò sbracciata e impudica; io/la cinsi di alloro da poeta: ella si risvegliò/lattante, latitante//L'amore era un gioco instabile; un gioco di /fonosillabe» ("Arremengada e impúdica se presentó el alba; yo/la ceñí con el laurel de los poetas; ella se despertó/lactante, rebelde.//El amor era un juego inestable, un juego/de fonosílabas»). E si rimpiange anche che la grazia 
sgrammaticata e mimeticamente frettolosa della popolaresca caduta della proposizione in "Se dalle tue lunghe agonie e dai miei brevi respiri/sorgesse un fiore; allora io correrei ringraziarti/rimboccherei la strada della bellezza. [...]», «Si de tus largas agonías y mis cortos suspiros/surgiera una flor; entonces correría a agradecerte/enhebrando la calle de tu belleza [...]», dove il gerundio dello spagnolo potrebbe, che so, anche giocare con la similarità del significante tra rimboccare e re-imboccare, ma non è facile: il trobar clus, spesso bifido, beffardo, di Rosselli se non lesina irrisioni e trappole ai propri stessi pronunciamenti gnomici, molte ne riserva anche a chi vi si accosti, insieme invitandolo ad una copula illogica, e respingendolo. Ed è spesso vivace Merlo nel reagire, tra fedeltà alle evidenze strutturanti più marcate (non tocca quasi le rumorose anafore che rinserrano i testi) e reinvenzione ritmica, quando l'urgenza traboccante ma avvitata del martellamento sonoro, percussivo, viene risaltata dall'iconismo metrico-sintattico nel testo tradotto, mediante i frequenti encabalgamientos inventati ex nibilo o resi più affilati e violenti rispetto alle inarcature, per tradizione leni, dell'italiano.

Rosselli, con un suo estremo testo critico tradotto è la vestale incaricata di presentare la raccolta Residenze Invernali dell'ottima Anedda, sempre per Igitur, nella versione dell'esperto Emilio Coco, responsabile di utili repertori in castigliano della lirica italiano tardonovecentesca. A Coco si deve pure il sobrio omaggio in postfazione a questo lirico nuovo. Il tutto è davvero squisito e rarefatto e sul resto spicca senz'altro l'eccellente aderenza alla discrezione pudica della voce dell'originale, la morbida fermezza a non cedere alla tentazione di alzare i decibel di una voce sommessa ed elegante (quanto Montale e quanto Luzi, ma nascosti, introflessi), che corteggia il silenzio, con mormorata sacralità achmatoviana. Forse con più acume percettivo si sarebbero potuti rendere quei lessemi di senso che sottilmente nei testi di Anedda trafiggono il silenzio o punteggiano la penombra. «Le nostre anime dovrebbero dormire/come dormono i corpi sottili/stare tra le lenzuola come un foglio/i capelli dietro le orecchie aperte/capaci di ascoltare. Carne/appuntita e fragile, cava/nel buio della stanza». Per un lirico che si pronuncia così è importante che lo scalpiccio sia tale, e così il fruscio, il tonfo, lo scintillio.

Piero Dal Bon

\section{Eugenio MONTALE}

\section{Las ocasiones}

Trad. de Carlos Vitale. Montblanc: Igitur/poesía, 2005

Le occasioni di Eugenio Montale finalmente godono di una traduzione allo spagnolo in volume autonomo ed esaustiva riguardo al corpus testuale. Il lavoro, merito dell'argentino Carlos Vitale, costituisce una resa fedele, letterale dei testi, che rispetta il formato dell'originale fino ai minimi dettagli e alla concinnità dello stile che lo definiscono (aspetti tipografici come il corsivo della poesia prelimina- re, Il balcone, la pagina in bianco che separa quest'ultima dal resto del volume e quella che separa i titoli delle singoli parti dal rispettivo corpus costituente; l'esatta disposizione sulla pagina, ecc.); vi sono inoltre le Note d'autore montaliane, tradotte ed inserite in calce al volume come nell'edizione italiana, mentre in apertura vi è un Prólogo di Alfonso Alegre Heitzmann, che tenta una breve ma attenta ed 